The Glasgow Mathematical Journal is the continuation of the Proceedings of the Glasgow Mathematical Association. It was founded in 1952 by the late Professor T. M. MacRobert and published with the support of the University Court of the University of Glasgow. The present name was adopted in 1967 and since 1987 the Journal has been owned by the Glasgow Mathematical Journal Trust, an independent charitable trust (Scottish Charity Number SC006017).

\title{
Editorial Policy
}

The Glasgow Mathematical Journal publishes original research papers in any branch of Pure or Applied Mathematics. Its policy is to feature a wide variety of research areas and it welcomes the submission of papers from all parts of the world. Selection for publication is on the basis of reports from specialist referees commissioned by the Subject Editors or Editor-in-Chief.

\section{Submission of Manuscripts}

The journal uses an online submission and refereeing system operated by ScholarOne Manuscripts. Details on how to submit a paper may be found at http://mc.manuscriptcentral.com/gmj.

When submitting a paper you will be asked to nominate a subject editor. The current subject editors and their areas of interest are as follows:

\section{SUBJECT EDITORS:}

$\begin{array}{llll}\text { Algebra } & \text { Dr. J. Grabowski } & \text { Analysis } & \text { Dr. V. Moroz } \\ \text { Algebra } & \text { Professor V. Mazorchuk } & \text { Analysis } & \text { Dr. N. Bez } \\ \text { Algebra } & \text { Professor P.H. Kropholler } & \text { Differential Geometry } & \text { Professor R. Bielawski } \\ \text { Algebraic Geometry } & \text { Professor G. Brown } & \text { Mathematical Physics } & \text { Dr. O. Chalykh } \\ \text { Algebraic Topology } & \text { Dr. A.J. Baker } & \text { Number Theory } & \text { Professor T. Dokchitser } \\ \text { Analysis } & \text { Dr. A. Skalski } & & \end{array}$

You should choose a subject editor closest to your field. Alternatively you may nominate the Editor-in-Chief, Prof. I.A.B.Strachan, who will assign a Subject Editor to deal with your submission.

During the submission process you will be asked to confirm that the paper is not being considered for publication elsewhere.

The Journal encourages the use of .pdf files. LaTeX and Postscript as well as other formats can be uploaded but these will be converted into .pdf format. Note that .dvi files cannot be converted. Care should be taken with this option, especially if non-standard macros are being used, and the .pdf version the system produces should be carefully checked before final submission.

The submission of hard copies of paper is discouraged. However if it is not possible for you to use the online system paper may be sent directly to: The Editorial Office, Glasgow Mathematical Journal, University of Glasgow, School of Mathematics and Statistics, University Gardens, Glasgow G12 80W, UK in hardcopy or on a 3.5 disk or by e-mail to: gmj@maths.gla.ac.uk. Most electronic formats, including .tex source with .dvi or .ps files, are acceptable. In the case of a hardcopy submission two copies should be sent. All submissions should be accompanied by the author's address, telephone and fax number, together with (if possible) an e-mail address. Upon acceptance of a paper, the author will be asked to transfer copyright to the Glasgow Mathematical Journal Trust.

\section{Layout of manuscripts}

Papers should be typed or word-processed in double spacing throughout, with wide margins and on one side of the paper. They should be prepared so as to conform to the practice in current issues of the Journal as regards general layout, references, tables and figures. Please avoid footnotes if possible. Papers must begin with an abstract of not more than 200 words, and they should list at least one AMS 2010 classification number or keyword. This information is also required before your manuscript can be uploaded. Instructions regarding unusual symbols or any typographical point that might cause confusion should be provided on a separate sheet. These may be uploaded as separate files. If special fonts are used, they should conform to the Journal's practice. Authors are reminded that they should retain a copy of anything submitted for publication since neither the Journal nor the publisher can accept liability for any loss.

\section{Illustrations}

Text figures must be suitable for direct photographic reproduction and supplied separate from the text but allocated clearly to sections of the text. Low resolution version may be uploaded at submission as separate files. Originals of figures should not be sent until the paper has been accepted. A list of captions for figures should be attached separately.

\section{References}

References should be placed at the end of the paper, arranged and numbered in alphabetical order of the author's names. Titles of journals should be abbreviated as in Mathematical Reviews. In the text, reference numbers should be enclosed in square brackets to distinguish them from formula numbers which should be quoted in round brackets. A reference to a book should give the author, title (in italics), edition, publisher, year of publication; e.g.

[1] P. T. Johnstone, Stone Spaces, Cambridge Studies in Advanced Math. no. 3 (Cambridge University Press, 1982).

A reference to an article should give the author, title of article, short title of periodical (in italics), series number (if any), volume number, year, and the beginning and end pages of the paper; e.g.

[2] R. Guralnick, T. Pentilla, C. E. Praeger and J. Saxl, Finite linear groups, Proc. London Math. Soc. (3) 78 (1999), $167-214$.

\section{Proofreading}

Prior to publication sets of proofs of each paper will be sent to authors. For papers with more than one author the proofs are sent to the first named author unless the Editors receive other instructions. Authors are asked to correct and return proofs promptly. Typographical or factual errors only may be changed at proof stage. The publisher reserves the right to charge authors for excessive corrections of nontypographical errors. No page charge is made.

\section{Offprints}

No paper offprints are provided, but the corresponding author will be sent the pdf of the published article. Print offprints may be purchased at extra cost at proof stage.

\section{Grants}

The Glasgow Mathematical Journal Trust Fund offers grants in support of mathematical projects and events of demonstrable benefit to mathematics in Scotland. Further details and application forms can be obtained from the Business Manager or from the World Wide Web at www.maths.gla.ac.uk/gmj/gmj-trust/.

This journal issue has been printed on FSC-certified paper and cover board. FSC is an independent, non-governmental, not-for-profit organization established to promote the responsible management of the world's forests. Please see www.fsc.org for information. 


\section{Glasgow \\ Mathematical \\ Journal}

\section{Volume 58 Part 3 September 2016}

\section{CONTENTS}

Abdenacer Makhlouf and Florin Panaite, Twisting operators, twisted tensor products and smash products for hom-associative algebras

Zenghui Gao and Zhaoyong Huang, Weak injective and weak flat complexes

Yanan Lin and Zhenqiang Zhou, Tilted Algebras and Crossed Products

Jan H. Fourie and Elroy D. Zeekoei, Classes of sequentially limited operators 573

Nathan Broomhead, John Christian Ottem and Artie Prendergast-Smith, Partially ample line bundles on toric varieties

A. N. Sergeev and A. P. Veselov, Jack-Laurent symmetric functions for special values of parameters 599

O. S. Sorokin, On the weak grothendieck group of a bezout ring

Silvano Delladio, A note on some topological properties of sets with finite perimeter 637

Nicolas Ricka, Equivariant Anderson duality and Mackey functor duality

Th. Theofanidis, Real hypersurfaces of non-flat complex space forms with generalized $\xi$ - parallel jacobi structure operator

Mikko Kemppainen, On vector-valued tent spaces and hardy spaces associated with non-negative self-adjoint operators

Tom Coates and Hiroshi Iritani, On the existence of a global neighbourhood

Julien Bichon, The group of bi-galois objects over the coordinate algebra of the frobenius-Lusztig kernel of SL(2)

Chris Cave and Dennis Dreesen, Equivariant compression of certain direct limit groups and amalgamated free products

Cambridge Journals Online For further information about this journal please go to the journal website at: journals.cambridge.org/gmj

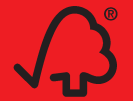

FSC

www.isc.org
MIX

Paper from responsible sources FSC ${ }^{\circledast} 007785$
CAMBRIDGE UNIVERSITY PRESS 